\title{
Remote ischaemic conditioning in percutaneous coronary intervention: a meta-analysis of randomised trials
}

\author{
Xiaowei Niu ${ }^{1}$, Jingjing Zhang ${ }^{1}$, De Chen ${ }^{1}$, Guozhen Wan ${ }^{1}$, Yiming Zhang ${ }^{2}$, Yali Yao ${ }^{2}$ \\ ${ }^{1}$ The First Clinical Medical School, Lanzhou University, Lanzhou, Gansu, China \\ ${ }^{2}$ Department of Cardiology, the First Hospital of Lanzhou University, Lanzhou, Gansu, China
}

Postep Kardiol Inter 2014; 10, 4 (38): 274-282

DOI: $10.5114 /$ pwki.2014.46771

\begin{abstract}
A bstract
Introduction: It remains uncertain whether remote ischaemic conditioning (RIC) using cycles of limb ischaemia-reperfusion as a conditioning stimulus benefits patients undergoing percutaneous coronary intervention ( $\mathrm{PCI})$.

Aim: We performed a meta-analysis toassessthe effect of RIC in PCI.

Material and methods: The PubMed, EMBASE, Web of Science, and CENTRAL databases were searched for randomised controlled trials (RCTs) comparing RIC with controls. The treatment effects were measured as a pooled odds ratio (OR), standardised mean difference (SMD), and corresponding $95 \%$ confidence intervals $(95 \% \mathrm{Cls})$ using random-effects models.

Results: Fourteen RCTs, including 2,301 patients, were analysed. Compared to the controls, RIC significantly reduced the cardiac enzyme levels (SMD $=-0.21 ; 95 \% \mathrm{Cl}:-0.39$ to $-0.04 ; p=0.015$; heterogeneity test, $l^{2}=75 \%$ ), and incidence of PCI-related myocardial infarction $(\mathrm{OR}=0.70 ; 95 \% \mathrm{Cl}, 0.51-0.98 ; p=0.037)$. There was a trend toward an improvement in the complete ST-segment resolution rate with $\mathrm{RIC}(\mathrm{OR}=1.83 ; 95 \% \mathrm{Cl}: 0.99-3.40 ; p=0.054)$. No significant difference could be detected between the two groups regarding the risk for acute kidney injury after PCl. Univariate meta-regression analysis suggested that the major source of significant heterogeneity was the PCI type (primary or non-emergent) for the myocardial enzyme levels (adjusted $R^{2}=0.44$ ). Subsequent subgroup analysis confirmed the results.

Conclusions: The present meta-analysis showed that RIC could confer cardioprotection for patients undergoing coronary stent implantation. Moreover, the decrease in the myocardial enzyme levels was more pronounced in the patients treated with primary PCI.
\end{abstract}

Key words: remote ischaemic preconditioning, remote ischaemic postconditioning, percutaneous coronary intervention, meta-analysis.

\section{Introduction}

Ischaemic heart disease is a leading cause of death worldwide [1]. Percutaneous coronary intervention (PCI) has played an important role in treating this disease in recent years. However, the process of blood reperfusion to the ischaemic myocardium can induce ischaemia-reperfusion injury (IRI) [2]. The phenomenon can paradoxically reduce the beneficial effects of $\mathrm{PCI}$ [2]. Several drugs and procedures to protect against IRI, such as the perioperative administration of adenosine, nicorandil, and therapeutic hypothermia, have been tested, but none of these interventions is completely effective [2]. Although classical conditioning by repeated intermittent balloon inflations may confer cardioprotection for patients undergoing $\mathrm{PCl}$, mechanic trauma to the vascular intima, increased procedure time, and risk of distal atheroma- tous embolisation into the microvasculature have limited their clinical applications [3, 4]. Thus, alternative strategies to further limit IRI are of major interest in the clinical setting [2-4].

Remote ischaemic conditioning (RIC) has become increasingly attractive because RIC can be achieved non-invasively by brief episodes of limb ischaemia with a blood pressure cuff or a pneumatic medical tourniquet $[3,4]$. Experimental studies have suggested that RIC protects against endothelial IRI in humans and triggers significant protection in numerous organs, not only the heart $[5,6]$. Some clinical studies have also been conducted to examine the effectiveness of RIC in patients undergoing PCI [7-20]. Nevertheless, not all trials have observed a favourable effect for RIC on myocardial injury based on cardiac enzyme levels. A previous meta-analysis of RIC

\section{Corresponding author:}

Dr Yali Yao, Department of Cardiology, the First Hospital of Lanzhou University, No. 1, Donggang West Road, Lanzhou, Gansu 730000, China, phone: +86 0931 8625200, fax: +86 931 8619797, e-mail: yaoyalifs@126.com

Received: 6.08 .2014 , accepted: 24.09.2014. 
in a broad $\mathrm{PCl}$ population (4 studies with 557 patients), by Yetgin et al. [21], reported no significant difference in myocardial injury biomarkers between RIC and controls $(p=0.36)$. The newly published trials, which could reduce the uncertainty regarding the treatment effects, have yet to be incorporated in a meta-analysis. Recently, two meta-analyses found that RIC before $\mathrm{PCI}$ reduced the incidence of $\mathrm{PCl}$-related myocardial infarction (PMI) $[22,23]$. However, they did not use a revised universal definition of PMI [24], which could limit extensive clinical application of RIC. Furthermore, the effect of RIC on renal protection in $\mathrm{PCl}$ has not been assessed in any previous meta-analysis.

\section{Aim}

Therefore, we performed a comprehensive metaanalysis to determine whether RIC provides myocardial and renal protection for patients undergoing $\mathrm{PCl}$. We also evaluated the potential factors that affect RIC performance.

\section{Material and methods}

We performed this meta-analysis according to the Preferred Reporting Items for Systematic Reviews and Meta-Analyses (PRISMA) statement [25] and the Cochrane Handbook guidelines [26]. All analyses were pre-specified, and the protocol for our study is registered in the international prospective register of systematic reviews (PROSPERO; registration number CRD42013006846, available from http://www.crd.york.ac.uk/PROSPERO/display_record.asp?ID=CRD42013006846).

\section{Selection criteria}

The following inclusion criteria were applied: (1) randomised clinical trials (RCTs) comparing RIC (defined as remote ischaemic pre-, per-, or post-conditioning) with controls (no conditioning) in patients undergoing non-emergent or primary $\mathrm{PCl}$ and (2) studies reporting data on any of the outcomes of interest (reported below). The exclusion criteria were (1) duplicated data and (2) sub-studies of the RCT.

\section{Search strategy}

Studies were identified by searching the PubMed, EMBASE, Web of Science, and Cochrane Central Register of Controlled Trials (CENTRAL) databases. This search was supplemented by scanning the reference lists of the eligible studies and recent review articles. No limits were placed on the language, date, or publication status. The major keywords and corresponding Medical Subject Headings were "remote ischaemic conditioning", "remote ischaemic preconditioning", "remote ischaemic postconditioning", "remote ischaemic perconditioning", and "percutaneous coronary intervention". The last search was performed on July 12, 2014.

\section{Study selection, data collection, and quality} assessment

Two independent investigators assessed the reports for eligibility in three screening stages at the title, abstract, and full-paper levels and then extracted data from the shortlisted studies on pre-specified forms. The following information was included: (1) the trial's design and inclusion criteria, (2) baseline patient and lesion characteristics, (3) features of the intervention and control arms, and (4) clinical outcomes. For missing or unclear information, we attempted to contact the original trial investigators by telephone or e-mail.

The same reviewers independently assessed the methodological quality of the eligible trials using the Jadad scale [27]. A score $\leq 2$ represents a low-quality study, and a score of at least 3 represents a high-quality study. All divergences were resolved by consensus or adjudication by a third reviewer.

\section{Study outcomes and definitions}

The primary endpoint chosen for this meta-analysis was myocardial enzyme levels, which included troponin $T$ $(\mathrm{TnT})$, troponin I (Tnl), and creatine kinase isoform-MB (CK-MB). The secondary endpoints were PMI, complete ST-segment resolution (CSTR), and acute kidney injury (AKI). The PMI was defined by an elevation in troponin $>5$ times the $99^{\text {th }}$ percentile in non-emergent $\mathrm{PCl}$ patients with a normal baseline value, according to the new definition [24]. cSTR was defined as ST-segment resolution $\geq 70 \%$ compared to the baseline measurement on the surface electrocardiogram after primary PCI [28]. The AKI was defined as a serum creatinine increase of $>25 \%$ over the baseline value or by more than $44.2 \mathrm{mmol} / \mathrm{l}$ after $\mathrm{PCl}$.

\section{Statistical analysis}

Two investigators examined the data from all identified studies. The standardised mean difference (SMD) and odds ratio (OR) (and their corresponding 95\% confidence intervals (Cls)) were calculated for the continuous or dichotomous outcome data, respectively. If the continuous data were reported as the median and interquartile range (IQR), the mean and standard deviation (SD) were estimated using the median and the estimated SD $(\mathrm{SD}=\mathrm{IQR} / 1.35)$ [26]. The number needed to treat (NNT) was calculated, when the pooled OR was statistically significant, as the inverse of pooled risk difference and $95 \% \mathrm{Cl}$. A random-effects model was used to account for the residual heterogeneity among trials and a more conservative summary estimate than the fixed effect analysis [26]. Statistical heterogeneity was evaluated with the Cochrane $\mathrm{Q}$ test and the $I^{2}$ statistic ( $p$ values $<0.1$ and $R^{2}$ values $>50 \%$ represented significant inconsistency) [26]. Meta-regression (a $p$ value of $<0.1$ was accepted) and subgroup analyses were performed to explore the potential sources of significant heterogeneity. To reduce the 
risk of over-fitting of the regression model, a minimum of ten studies were set to identify each influential factor [26]. Sensitivity analyses were conducted to assess the consistency of the main results by testing whether consecutively deleting each trial would influence the overall estimates for the myocardial enzyme levels. A study was considered to be influential if its exclusion changed the effect estimate by at least 20\% [29]. All findings were also repeated using a fixed-effect model, and only the data from high-quality studies were analysed. Funnel plot and Egger's tests were used to evaluate the presence of publication bias regarding our primary outcome. The results were statistically significant with a two-sided $p<0.05$. Statistical computations were performed with STATA 11.0 (Stata Corp., College Station, Texas, USA).

\section{Results}

\section{Eligible studies}

From a total of 116 potentially relevant publications (Figure 1), 14 RCTs fulfilled the inclusion criteria and were selected [7-20]. Among 2,301 patients enrolled from 10 countries, 1,230 were randomised to RIC and 1,071 to the control group. Eleven studies were performed in patients undergoing non-emergency $\mathrm{PCl}$, and 3 studies used primary $\mathrm{PCl}$ patients. The RIC was performed by inflating a cuff placed on the arm or leg to $200 \mathrm{~mm} \mathrm{Hg}$ or above the systolic pressure. In 10 studies, RIC was induced before the expected period of ischaemia (preconditioning). Nine studies had $\geq 30$-minute duration of
RIC, which was calculated by multiplying the duration of ischaemia/reperfusion per cycle by the number of cycles. For myocardial biomarkers, troponin I or T was used in 13 studies, and CK-MB was used in one. The biomarkers were measured using conventional assays in all trials except two, which used high-sensitivity assays. The mean patient age in the individual trials ranged from 54 to 69 years, and most were male. The percentages of patients with diabetes, hypertension, dyslipidaemia, and previous MI ranged from $9 \%$ to $100 \%, 0$ to $82 \%, 17 \%$ to $80 \%$, and 0 to $55 \%$, respectively. $\beta$-Blockers and glycoprotein $\mathrm{llb} /$ Illa inhibitors were used in most of the studies. Coronary angiography showed that the proportion of left anterior descending (LAD) culprit arteries among patients varied from $25 \%$ to $100 \%$. In terms of quality, 11 studies had a Jadad score $\geq 3$ points, and the remaining three scored $<3$ points. Tables I and II show the characteristics and demographic data for these studies.

\section{Quantitative outcomes}

The meta-analysis of 14 RCTs showed that RIC significantly reduced the postoperative myocardial enzyme levels in a broad $\mathrm{PCl}$ population (SMD $=-0.21 ; 95 \% \mathrm{Cl}$ : -0.39 to $-0.04 ; p=0.015)$ with significant heterogeneity $\left(I^{2}=75 \%\right)$ (Figure 2).

During non-emergent $\mathrm{PCl}, \mathrm{PMI}$ was reported in 237 of 708 patients $(33.5 \%)$ in the RIC arm compared to 252 of 585 patients $(43.1 \%)$ in the control arm of the randomized trials. The OR for PMI for the RIC group compared to the control group was 0.70 (95\% Cl: 0.51-0.98;

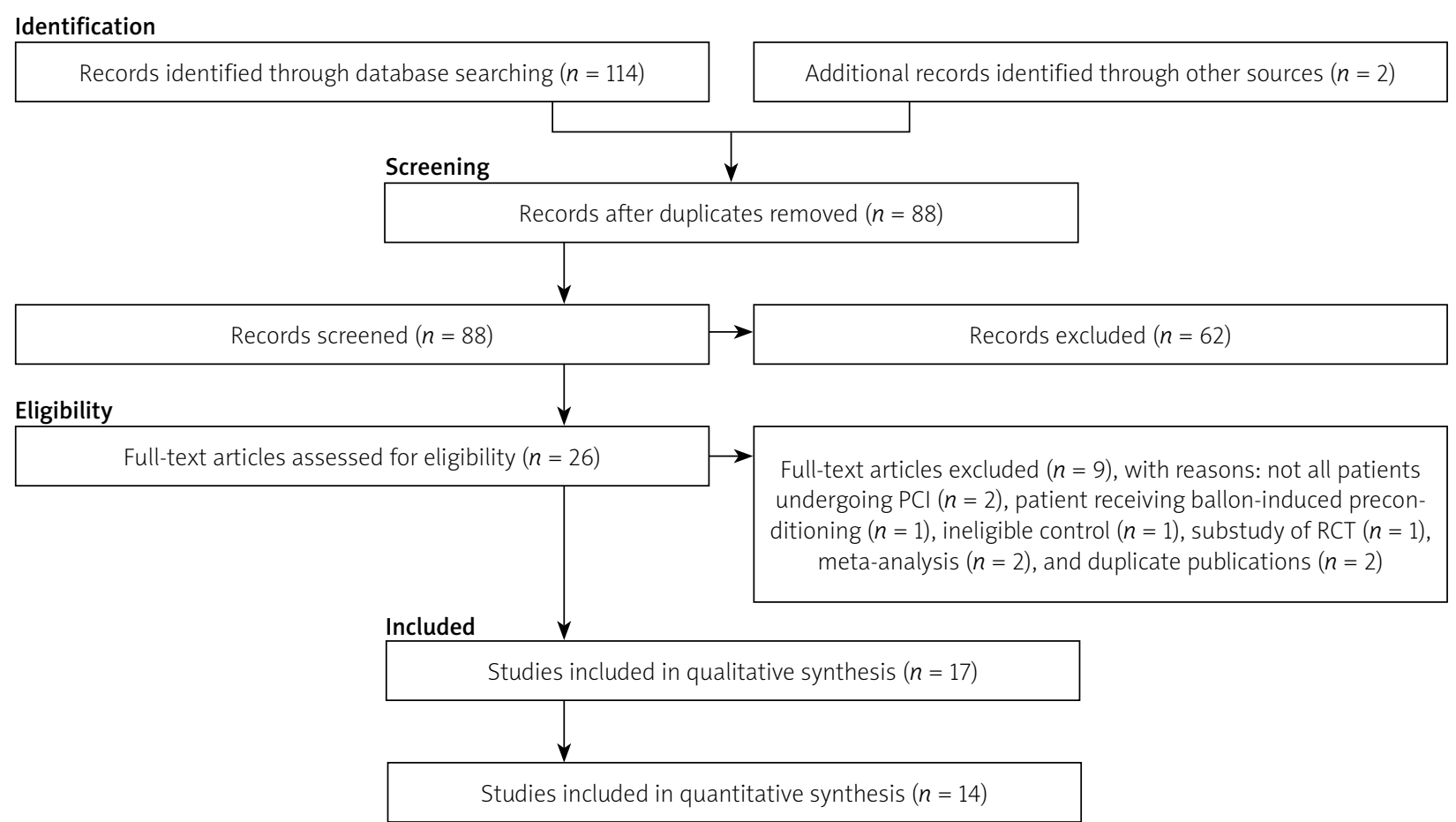

Figure 1. Flow diagram of the review process, according to the PRISMA statement

PRISMA - Preferred Reporting Items for Systematic reviews and Meta-Analyses, PCI-percutaneous coronary intervention, RCT-randomised controlled trial 

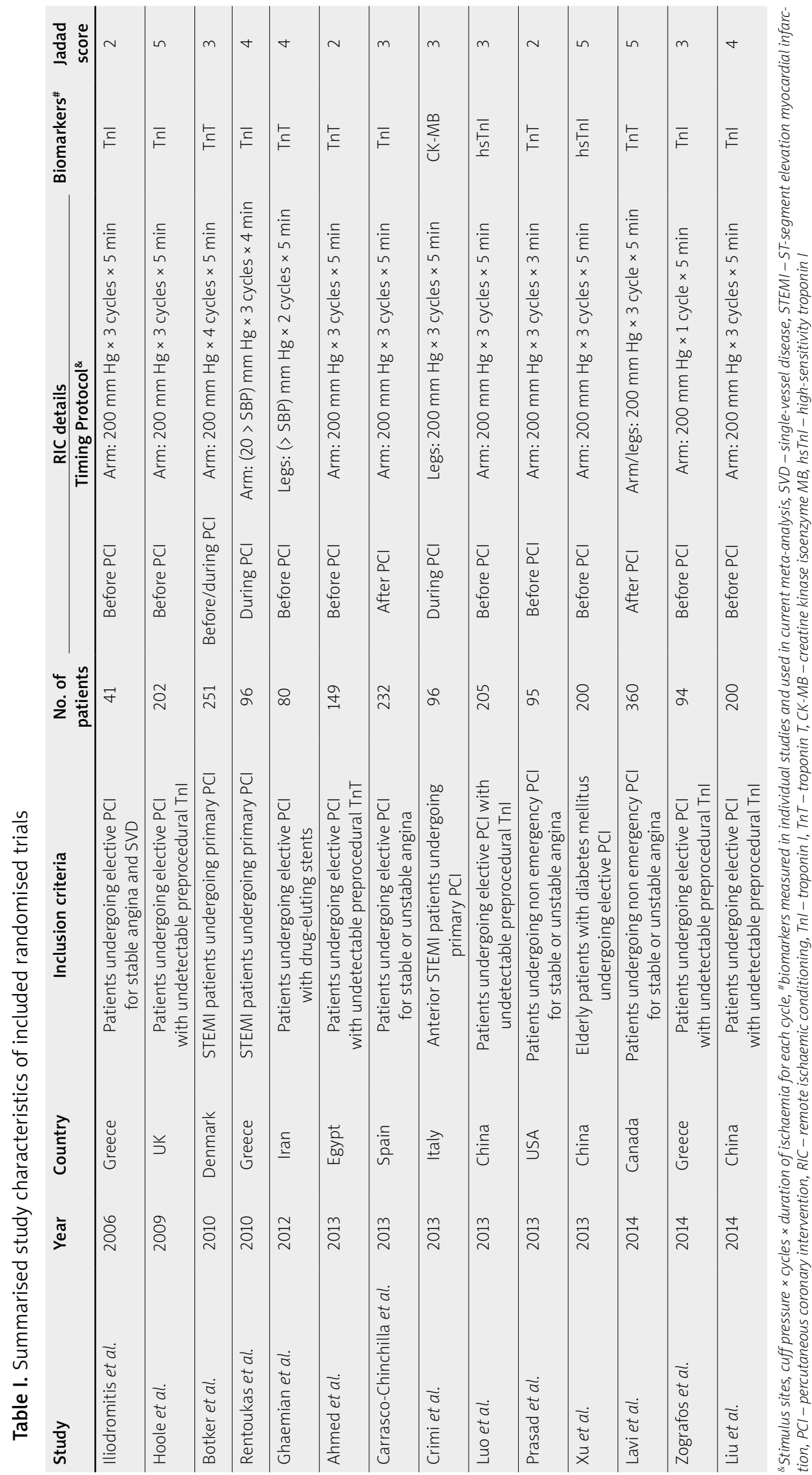
Table II. Summarised demographic data of included randomised trials

\begin{tabular}{|c|c|c|c|c|c|c|c|c|c|}
\hline Study & $\begin{array}{c}\text { Age } \\
\text { [years] }\end{array}$ & $\begin{array}{c}\text { Male } \\
(\%)\end{array}$ & $\begin{array}{l}\text { Diabetes } \\
(\%)\end{array}$ & $\begin{array}{l}\text { Hypertension } \\
\text { (\%) }\end{array}$ & $\begin{array}{c}\text { Dyslipidaemia } \\
\text { (\%) }\end{array}$ & $\begin{array}{l}\text { Previous } \\
\text { MI (\%) }\end{array}$ & $\begin{array}{c}\beta \text {-Blockers } \\
(\%)\end{array}$ & $\begin{array}{c}\text { GP Ilb/IIla } \\
\text { inhibitors } \\
(\%)\end{array}$ & $\begin{array}{c}\text { LAD } \\
\text { lesion } \\
(\%)\end{array}$ \\
\hline Iliodromitis et al. & 62 & NR & 34 & 0 & 80 & 0 & 71 & 41 & 56 \\
\hline Hoole et al. & 63 & 78 & 22 & 51 & NR & 55 & 79 & 0 & 42 \\
\hline Botker et al. & 63 & 76 & 9 & 31 & 17 & 0 & NR & 85 & 41 \\
\hline Rentoukas et al. & 63 & 61 & 31 & 46 & 44 & 15 & 96 & NR & NR \\
\hline Ghaemian et al. & 60 & 48 & 36 & 49 & 74 & 9 & 81 & 0 & 66 \\
\hline Ahmed et al. & 54 & 87 & 52 & 64 & 66 & NR & NR & 23 & 40 \\
\hline Carrasco-Chinchilla et al. & 65 & 68 & 42 & 76 & 62 & 0 & 83 & 4 & 55 \\
\hline Crimi et al. & 58 & 88 & 11 & 52 & 31 & 11 & 16 & 96 & 100 \\
\hline Luo et al. & 59 & 76 & 28 & 66 & NR & 21 & 82 & 51 & 51 \\
\hline Prasad et al. & 66 & 83 & 27 & 78 & 74 & 28 & 74 & 49 & 53 \\
\hline Xu et al. & 69 & 68 & 100 & 64 & NR & 23 & 80 & 47 & 35 \\
\hline Lavi et al. & 64 & 74 & 31 & 70 & 66 & 43 & NR & 25 & 40 \\
\hline Zografos et al. & 61 & 88 & 38 & 82 & 72 & 20 & 82 & NR & 46 \\
\hline Liu et al. & 58 & 54 & 36 & 63 & $N R$ & $N R$ & 81 & 0 & 25 \\
\hline
\end{tabular}

MI-myocardial infarction, GP-glycoprotein, LAD - left anterior descending, NR - not reported

Myocardial enzyme levels

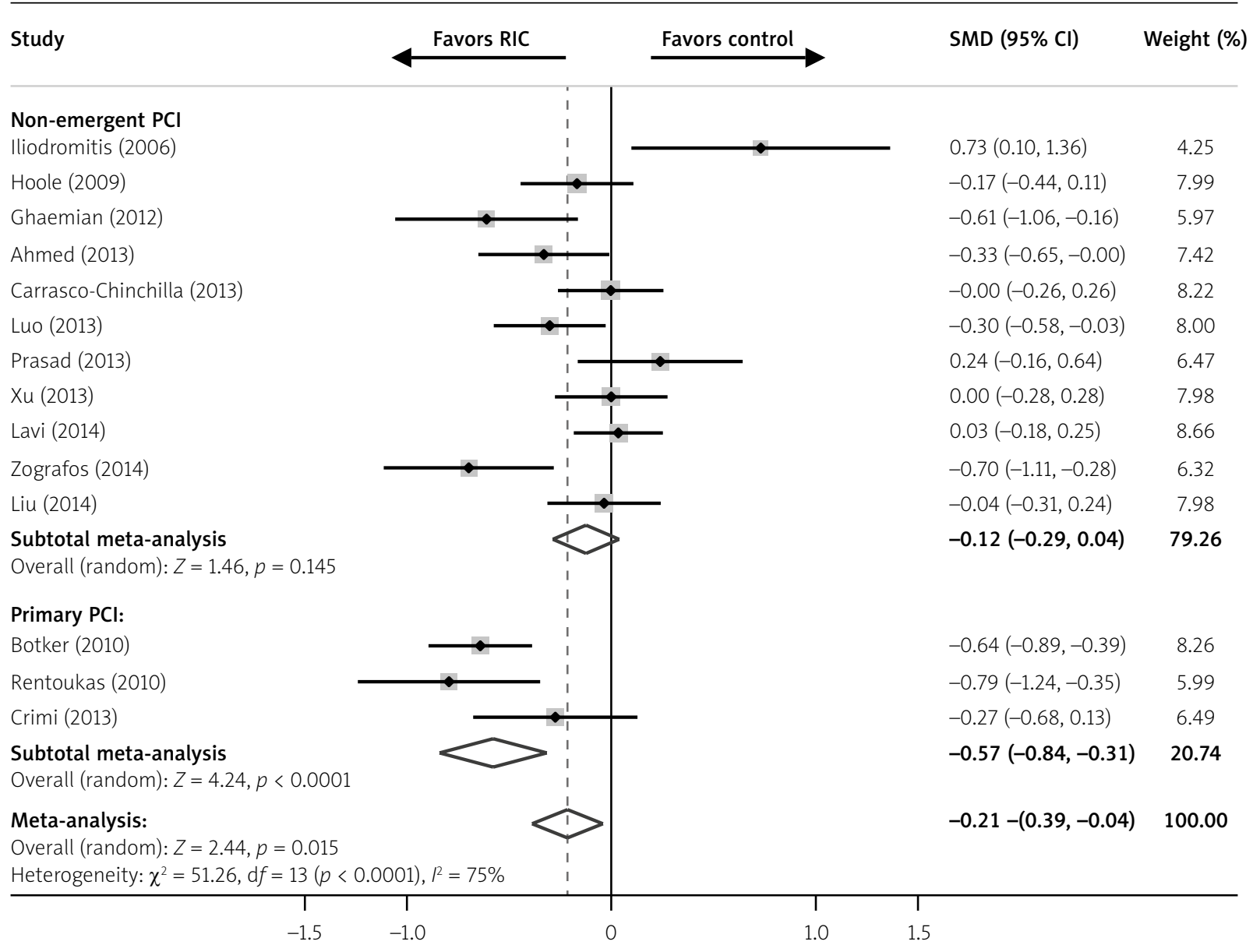

Figure 2. Forest plot for myocardial enzyme levels, expressed as standardised mean differences (SMDs) with $95 \% \mathrm{Cls}$

RIC-remote ischaemic conditioning, Cl-confidence interval, df-degree of freedom 
PCl-related myocardial infarction

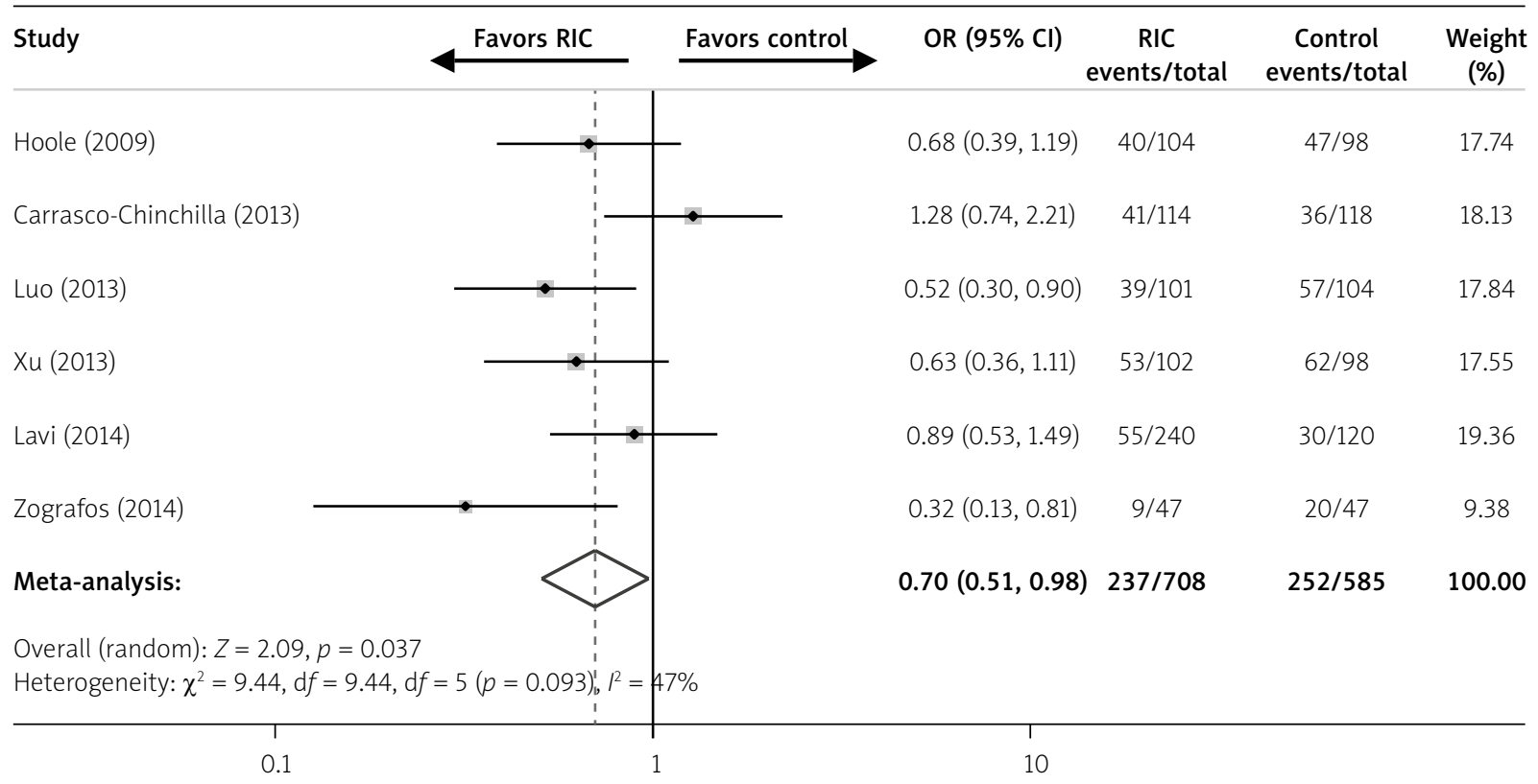

Figure 3. Forest plot for $\mathrm{PCl}$-related myocardial infarction with or without remote ischaemic conditioning (RIC) in patients undergoing non-emergent $\mathrm{PCl}$

$O R$ - odds ratio, $\mathrm{Cl}$ - confidence interval, $d f$ - degree of freedom

$p=0.037 ; l^{2}=47 \%$ ) (Figure 3 ). The NNT was 12 (7 to 203), in other words 12 patients who would need to be treated with the RIC to prevent one PMI.

Among primary $\mathrm{PCl}$ patients randomised to RIC, 127 of 207 (61.4\%) had cSTR compared to 104 of 203 (51.2\%) patients who were randomised to the control group $\left(\mathrm{OR}=1.83 ; 95 \% \mathrm{Cl}: 0.99-3.40 ; p=0.054 ; P^{2}=33 \%\right)$ (Figure 4).
In the four trials that reported AKI endpoints during $\mathrm{PCI}[12,14,17,18]$, the incidence of AKI was $4.8 \%$ (26 of 547 ) in the RIC group and $5.0 \%$ (21 of 420 ) in the control group. No significant difference could be detected between the two groups regarding the risk for AKI (0.89 $\left.(0.48-1.64), p=0.701 ; I^{2}=0 \%, p_{\text {heterogeneity }}=0.65\right)$.

Complete ST-segment resolution

\begin{tabular}{|c|c|c|c|c|c|}
\hline Favors control & Favors RIC & OR $(95 \% \mathrm{Cl})$ & $\begin{array}{c}\text { RIC } \\
\text { events/total }\end{array}$ & $\begin{array}{c}\text { Control } \\
\text { events/total }\end{array}$ & $\begin{array}{c}\text { Weight } \\
(\%)\end{array}$ \\
\hline Botker (2010) & $\frac{1}{1}$ & $1.27(0.74,2.18)$ & $91 / 126$ & $84 / 125$ & 54.49 \\
\hline Rentoukas (2010) & & $2.33(0.82,6.66)$ & $24 / 33$ & $16 / 30$ & 25.26 \\
\hline Crimi (2013) & & -3.67 (1.09, 12.35) & $12 / 48$ & $4 / 48$ & 20.25 \\
\hline $\begin{array}{l}\text { Meta-analysis: } \\
\text { Overall (random): } Z=1.93, p=0.054 \\
\text { Heterogeneity: } \chi^{2}=2.99, \mathrm{~d} f=2(p=0.225), I^{2}=33 \%\end{array}$ & & $1.83(0.99,3.40)$ & $127 / 207$ & $104 / 203$ & 100.00 \\
\hline $\begin{array}{c}1 \\
0.05\end{array}$ & & $\begin{array}{l}1 \\
15\end{array}$ & & & \\
\hline
\end{tabular}

Figure 4. Forest plot for complete ST-segment resolution with or without remote ischaemic conditioning (RIC) in patients undergoing primary $\mathrm{PCl}$

$O R$ - odds ratio, $\mathrm{Cl}$ - confidence interval, $d f$-degree of freedom 
Table III. Sensitivity analyses

\begin{tabular}{lcccc} 
& Myocardial enzyme levels & PMI & cSTR & AKI \\
\hline \begin{tabular}{l} 
Statistical model: \\
\hline Fixed-effect model
\end{tabular} & $-0.20(-0.28,-0.11)^{\star}$ & $0.72(0.57,0.91)^{\star}$ & $1.64(1.05,2.56)^{\star}$ & $0.89(0.48,1.62)$ \\
\hline Random-effects model & $-0.20(-0.39,-0.04)^{\star}$ & $0.70(0.51,0.98)^{\star}$ & $1.83(0.99,3.40)$ & $0.89(0.48,1.64)$ \\
\hline Study quality & & & & $0.89(0.48,1.64)$ \\
\hline High study quality\& & $-0.23(-0.32,-0.14)^{\star}$ & $0.70(0.51,0.98)^{\star}$ & $1.83(0.99,3.40)$ &
\end{tabular}

The pooled estimates are reported as standardised mean difference, odds ratio, and corresponding $95 \%$ confidence intervals. PMI-PCI-related myocardial infarction, CSTR - complete ST-segment resolution, AKI - acute kidney injury, \&Removing Jadad score $\leq 2$ studies [7, 13, 15], *significant comparisons ( $p<0.05$ )

\section{Potential sourcesof heterogeneity and subgroup analysis}

The random-effect univariate meta-regression analysis for the myocardial enzyme levels in a broad $\mathrm{PCl}$ population was conducted to explore the potential sources of heterogeneity. Data on the country (Europe or non-Europe), $\mathrm{PCl}$ type (primary or non-emergent), duration of the $\mathrm{RIC}$ protocol ( $<30$ or $\geq 30 \mathrm{~min}$ ), timing of the intervention (preconditioning or non-preconditioning), limb used (arm or leg), age, sex (\% male), diabetes (\%), hypertension (\%), previous MI (\%), dyslipidaemia (\%), $\beta$-blockers use (\%), glycoprotein IIb/IIla inhibitor use (\%), and presence of an LAD lesion (\%) were included. As a result, $\mathrm{PCl}$ type was the major heterogeneity source identified (coefficient $=-0.45$, $p=0.057$, adjusted $R^{2}=0.44$ ). Subsequent subgroup analysis was then performed based on the $\mathrm{PCl}$ type. Compared to the non-emergent $\mathrm{PCl}$ subgroup, the primary $\mathrm{PCl}$ subgroup showed a significant reduction in the myocardial enzyme levels (SMD, -0.57 ( $p<0.0001)$ vs. $-0.12(p=0.145)$; $p<0.0001$ for the subgroup difference) (Figure 2).

\section{Sensitivity and publication bias}

The sensitivity analysis deleting each trial in turn found that no single study significantly altered the summary SMD for the cardiac biomarkers. The overall treatment effect for the RIC remained consistent for each endpoint using either a fixed- or random-effects model and only analysing the data from high-quality studies (Table III). In addition, we found that compared to the controls, RIC significantly reduced the cardiac enzyme levels $(-0.13(-0.23,-0.03), p=0.01)$ in patients undergoing non-emergent $\mathrm{PCl}$ when pooling data from eight studies with low risk of bias.

Visual inspection of the funnel plot for the cardiac biomarkers did not reveal an apparent asymmetry; this finding was supported by Egger's test $(p=0.71)$.

\section{Discussion}

In this meta-analysis of 14 randomised trials involving 2,301 patients, the main findings can be summarised as follows: (1) Compared to coronary intervention alone, RIC significantly reduced the myocardial enzyme levels and risk of $\mathrm{PCl}$-related $\mathrm{MI}$ in patients after $\mathrm{PCl}$. Furthermore, the decrease in the myocardial injury biomarkers was more pronounced among the patients treated with primary $\mathrm{PCl}$. (2) Although the statistical significance of the difference was marginal, there was a trend toward an improvement in the cSTR rate with RIC. In fact, the pooled analysis based on the fixed-effect model showed that RIC significantly improved the CSTR outcomes.

The RIC is an attractive strategy because this simple, inexpensive, and well-tolerated technique can be easily implemented in a busy PCl centre. The actual cardioprotective mechanism for RIC is not fully understood. Many researchers believe that the process is multifactorial and involves the regulation of neural reflexes; the release of humoral factors, such as adenosine and opioids; the elaboration of endogenous myocardial mediators including nitric oxide and free radicals; and the activation of aK(ATP)-channel [4]. Furthermore, the results from randomised studies showed that ischaemic conditioning had a beneficial platelet inhibitory and anti-inflammatory effect, which might stabilise vulnerable plaques [30, 31]. The RIC induced before PCI for myocardial infarction was associated with a significant and sustained improvement of endothelial function [31]. Overall, this evidence from animal and clinical studies may partly explain the effect of RIC in PCl.

Studies have shown that single time-point assessment and peak levels of cardiac biomarkers are significantly correlated with infarct size and early left ventricular function, which are closely related to prognosis, in patients with ST-segment elevation myocardial infarction (STEMI) [32]. Data from cardiac magnetic resonance-delayed enhancement imaging have confirmed that the release of cardiac biomarkers after elective $\mathrm{PCl}$ is indicative of new irreversible myocardial injury, and the magnitude of this injury highly correlates with the extent of the elevation of biomarker levels post-PCI [33]. The increases in cardiac enzymes are associated with poor long-term outcomes after elective $\mathrm{PCI}[24,34]$. In our study, we detected significant reductions in the myocardial biomarkers among the broad $\mathrm{PCl}$ population, and such protective effects were more significant in STEMI patients undergoing primary $\mathrm{PCl}$. The reason for the latter finding was that the 
contribution of ischaemia-reperfusion damage to cardiac injury varied with the clinical setting. For instance, in the setting of primary $\mathrm{PCl}$, the cardiac injury was the sum of IRI caused by ischaemic cell death and rapid recanalisation/reperfusion of an occluded epicardial artery. Therefore, STEMI patients represent a high-risk population for the development of lethal IRI and may have greater increases in myocardial injury biomarkers [35]. In this setting, the potential benefit of RIC would be amplified. However, the myocardial injury during non-emergent $\mathrm{PCl}$ was relatively minimal because of a lack of acute lethal IRI and was mostly caused by side branch loss and distal embolisation of the coronary artery during balloon inflation or stent deployment, followed by spontaneous lysis and reperfusion [21, 35]. Although our results in the subgroup analysis showed that RIC did not reduce cardiac enzyme levels in patients undergoing non-emergent $\mathrm{PCl}$, this benefit reached statistical significance after exclusion of low-quality trials. Importantly, we found that post-PCl elevation of troponin more than 5-times the baseline level, which specifically identifies $\mathrm{PCl}$ as a cause of myocardial infarction (PMI) in the guidelines, was less frequent in the RIC group than in the control group. Thus, considering that peri-procedural myocardial injury and infarction are common findings (up to 30\%) and are associated with worse prognosis [34], it is likely that limiting injuries by using RIC is beneficial to patients undergoing non-emergent $\mathrm{PCl}$.

Microvascular obstruction is an irreversible form of IRI, which results in the death of both endothelial cells and cardiomyocytes [36]. cSTR has been proposed as an electrocardiographic index of microvascular reperfusion, and CSTR yields prognostic information in addition to the data provided by the myocardial blush grade [36]. A relationship between cSTR and subsequent mortality has also been well described in previous studies [28, 36, 37]. Although the current study failed to show a statistically significant improvement in the CSTR rate after primary $\mathrm{PCl}$ in the RIC group compared to the control group, there was an obvious trend toward RIC and a positive result based on the fixed-effect model. This finding may support the active use of RIC for high-risk coronary no-reflow patients after primary $\mathrm{PCl}$.

Contrast-induced nephropathy remains a common complication after $\mathrm{PCl}$. The results from a recent randomised trial have suggested that RIC before elective coronary angiography can prevent contrast-induced AKI in patients with renal dysfunction [38]. In our study, the risk of AKI showed no difference between the control and the RIC group. The potential reasons for this disparity were inadequate sample size. Indeed, the power calculation in all included trials was not based on the incidence of $\mathrm{AKI}$ after $\mathrm{PCl}$. Further investigations will be required to establish the effect of RIC on prevention of AKI.
Our study has several limitations. First, because this meta-analysis is not based on patient-level data, our study shares the possible shortcomings of the original articles. We did not conduct subset analyses of the patients with diabetes, hypertension, and LAD lesions because the meta-regression analysis did not show a significant effect of these covariates on the myocardial enzyme levels. Second, we pooled the data for biomarkers at various time-points, but the conclusion was based on a random-effects model to compensate for a certain degree of heterogeneity. Given that troponin has a peak level at around $24 \mathrm{~h}$ after myocardial necrosis, the troponin level at $16 \mathrm{~h}$ post- $\mathrm{PCl}$ was probably closer to the "actual" peak level. Third, the numbers of trials and patients included in some analyses were relatively small, so the results should be interpreted with caution. Finally, although our meta-regression analysis indicated that the duration of RIC, the use of the upper or lower limb, and the time difference between the conditioning stimulus and $\mathrm{PCl}$ did not affect the outcome, the lack of a standard protocol may potentially influence the positive cardioprotection effects of RIC. An adequately powered trial is merited to identify the optimal type and algorithm for the conditioning stimulus.

\section{Conclusions}

The present meta-analysis demonstrated that RIC, using repeated brief episodes of limb ischaemia, can confer cardioprotection for patients undergoing primary or non-emergent $\mathrm{PCl}$. Moreover, the decrease in the myocardial enzyme levels was more pronounced in the patients treated with primary $\mathrm{PCI}$.

\section{References}

1. World Health Organization. The top 10 causes of death. Available at: http://www.who.int/mediacentre/factsheets/fs310/en/. Updated July 2013.

2. Yellon DM, Hausenloy DJ. Myocardial reperfusion injury. N Engl J Med 2007; 357: 1121-35.

3. Bell RM, White SK, Yellon DM. Remote ischaemic conditioning: building evidence of efficacy. Eur Heart J 2013; 35: 138-40.

4. Hausenloy DJ, Yellon DM. The therapeutic potential of ischaemic conditioning: an update. Nat Rev Cardiol 2011; 8: 619-29.

5. Kharbanda RK, Mortensen UM, White PA, et al. Transient limb ischemia induces remote ischaemic preconditioning in vivo. Circulation 2002; 106: 2881-3.

6. Candilio L, Malik A, Hausenloy DJ. Protection of organs other than the heart by remote ischaemic conditioning. J Cardiovasc Med (Hagerstown) 2013; 14: 193-205.

7. Ahmed RM, Mohamed EHA, Ashraf M, et al. Effect of remote ischaemic preconditioning on serum troponin $\mathrm{T}$ level following elective percutaneous coronary intervention. Catheter Cardiovasc Interv 2013; 82: E647-53.

8. Bøtker HE, Kharbanda R, Schmidt MR, et al. Remote ischaemic conditioning before hospital admission, as a complement to angioplasty, and effect on myocardial salvage in patients with 
acute myocardial infarction: a randomised trial. Lancet 2010; 375: 727-34.

9. Carrasco-Chinchilla F, Muñoz-García AJ, Domínguez-Franco A, et al. Remote ischaemic postconditioning: does it protect against ischaemic damage in percutaneous coronary revascularisation? Randomised placebo-controlled clinical trial. Heart 2013; 99: 1431-7.

10. Crimi G, Pica S, Raineri C, et al. Remote ischaemic post-conditioning of the lower limb during primary percutaneous coronary intervention safely reduces enzymatic infarct size in anterior myocardial infarction: a randomized controlled trial. JACC Cardiovasc Interv 2013; 6: 1055-63.

11. Ghaemian A, Nouraei SM, Abdollahian F, et al. Remote ischaemic preconditioning in percutaneous coronary revascularization: a double-blind randomized controlled clinical trial. Asian Cardiovasc Thorac Ann 2012; 20: 548-54.

12. Hoole SP, Heck PM, Sharples L, et al. Cardiac remote ischaemic preconditioning in coronary stenting (CRISP Stent) study: a prospective, randomized control trial. Circulation 2009; 119: 820-7.

13. Iliodromitis EK, Kyrzopoulos S, Paraskevaidis IA, et al. Increased $C$ reactive protein and cardiac enzyme levels after coronary stent implantation. Is there protection by remote ischaemic preconditioning? Heart 2006; 92: 1821-6.

14. Luo SJ, Zhou YJ, Shi DM, et al. Remote ischaemic preconditioning reduces myocardial injury in patients undergoing coronary stent implantation. Can J Cardiol 2013; 29: 1084-9.

15. Prasad A, Gössl M, Hoyt J, et al. Remote ischaemic preconditioning immediately before percutaneous coronary intervention does not impact myocardial necrosis, inflammatory response, and circulating endothelial progenitor cell counts: a single center randomized sham controlled trial. Catheter Cardiovasc Interv 2012; 81: 930-6.

16. Rentoukas I, Giannopoulos G, Kaoukis A, et al. Cardioprotective role of remote ischaemic periconditioning in primary percutaneous coronary intervention: enhancement by opioid action. JACC Cardiovasc Interv 2010; 3: 49-55.

17. Xu X, Zhou Y, Luo S, et al. Effect of remote ischaemic preconditioning in the elderly patients with coronary artery disease with diabetes mellitus undergoing elective drug-eluting stent implantation. Angiology 2014; 65: 660-6.

18. Lavi S, D’Alfonso S, Diamantouros P, et al. Remote ischaemic postconditioning during percutaneous coronary interventions: remote ischaemic postconditioning-percutaneous coronary intervention randomized trial. Circ Cardiovasc Interv 2014; 7: 225-32.

19. Zografos TA, Katritsis GD, Tsiafoutis I, et al. Effect of one-cycle remote ischaemic preconditioning to reduce myocardial injury during percutaneous coronary intervention. Am J Cardiol 2014; 113: 2013-7.

20. Liu Z, Wang YL, Xu D, et al. Late remote ischaemic preconditioning provides benefit to patients undergoing elective percutaneous coronary intervention. Cell Biochem Biophys 2014; doi: 10.1007/s12013-014-9936-1.

21. Yetgin T, Manintveld OC, Boersma E, et al. Remote ischaemic conditioning in percutaneous coronary intervention and coronary artery bypass grafting. Circ J 2012; 76: 2392-404.

22. D’Ascenzo F, Moretti C, Omede P, et al. Cardiac remote ischaemic preconditioning reduces periprocedural myocardial infarction for patients undergoing percutaneous coronary interventions: a meta-analysis of randomised clinical trials. Eurolntervention 2014; 9: 1463-71.
23. Zografos TA, Katritsis GD, Katritsis DG. Remote ischaemic preconditioning reduces peri-procedural myocardial injury in elective percutaneous coronary intervention: a meta-analysis. Int J Cardiol 2014; 173: 530-2.

24. Thygesen K, Alpert JS, Jaffe AS, et al. Third universal definition of myocardial infarction. Eur Heart J 2012; 33: 2551-67.

25. Moher D, Liberati A, Tetzlaff J, et al. Preferred reporting items for systematic reviews and meta-analyses: the PRISMA statement. BMJ 2009; 339: b2535.

26. Higgins J, Green S. Cochrane handbook for systematic reviews of interventions version 5.1. 0 [updated March 2011].The Cochrane Collaboration 2011. Available from www.cochrane-handbook.org.

27. Jadad AR, Moore RA, Carroll D, et al. Assessing the quality of reports of randomized clinical trials: is blinding necessary? Control Clin Trials 1996; 17: 1-12.

28. Schröder R. Prognostic impact of early ST-segment resolution in acute ST-elevation myocardial infarction. Circulation 2004; 110 : e506-10.

29. Niu X, Yang C, Chen D, et al. Impact of drug-eluting stents with different coating strategies on stent thrombosis: a meta-analysis of 19 randomized trials. Cardiol J 2014; doi:10.5603/ CJ.a2014.0002.

30. Lee TM, Lin MS, Tsai CH, et al. Effect of ischaemic preconditioning on regional release of inflammatory markers. Clin Sci (Lond) 2005; 109: 267-76.

31. Manchurov V, Ryazankina N, Khmara T, et al. Remote ischaemic preconditioning and endothelial function in patients with acute myocardial infarction and primary PCI. Am J Med 2014; 127: 670-3.

32. Chia S, Senatore F, Raffel OC, et al. Utility of cardiac biomarkers in predicting infarct size, left ventricular function, and clinical outcome after primary percutaneous coronary intervention for ST-segment elevation myocardial infarction. JACC Cardiovasc Interv 2008; 1: 415-23.

33. Selvanayagam JB, Porto I, Channon K, et al. Troponin elevation after percutaneous coronary intervention directly represents the extent of irreversible myocardial injury: insights from cardiovascular magnetic resonance imaging. Circulation 2005; 111: 1027-32.

34. Testa L, Van Gaal WJ, Biondi Zoccai GG, et al. Myocardial infarction after percutaneous coronary intervention: a meta-analysis of troponin elevation applying the new universal definition. QJM 2009; 102: 369-78.

35. Prasad A, Herrmann J. Myocardial infarction due to percutaneous coronary intervention. N Engl J Med 2011; 364: 453-64.

36. Brener SJ, Dizon JM, Mehran R, et al. Complementary prognostic utility of myocardial blush grade and ST-segment resolution after primary percutaneous coronary intervention: analysis from the HORIZONS-AMI trial. Am Heart J 2013; 166: 676-83.

37. Masoomi M, Samadi S, Sheikhvatan M. Thrombolytic effect of streptokinase infusion assessed by ST-segment resolution between diabetic and non-diabetic myocardial infarction patients. Cardiol J 2012; 19: 168-73.

38. Er F, Nia AM, Dopp H, et al. Ischaemic preconditioning for prevention of contrast medium-induced nephropathy: randomized pilot RenPro Trial (Renal Protection Trial). Circulation 2012; 126 : 296-303. 